\title{
In Situ Evaluation of the Influence of Interstitial Oxygen on the Elastic Modulus of $\mathrm{La}_{2} \mathrm{NiO}_{4}$
}

\author{
Yuta Kimura ${ }^{1, *}$, Takashi Nakamura ${ }^{1}$, Koji Amezawa $^{1}$, Keiji Yashiro $^{2} \odot$ and Tatsuya Kawada ${ }^{2}$ \\ 1 Institute of Multidisciplinary Research for Advanced Materials, Tohoku University, 2-1-1 Katahira, Aoba-ku, \\ Sendai 980-8577, Miyagi, Japan; takashi.nakamura.e3@tohoku.ac.jp (T.N.); \\ koji.amezawa.b3@tohoku.ac.jp (K.A.) \\ 2 Graduate School of Environmental Studies, Tohoku University, 6-6-04, Aramaki Aoba Aoba-ku, \\ Sendai 980-8579, Miyagi, Japan; keiji.yashiro.d7@tohoku.ac.jp (K.Y.); tatsuya.kawada.b7@tohoku.ac.jp (T.K.) \\ * Correspondence: yuta.kimura.b2@tohoku.ac.jp
}

Citation: Kimura, Y.; Nakamura, T.; Amezawa, K.; Yashiro, K.; Kawada, T. In Situ Evaluation of the Influence of Interstitial Oxygen on the Elastic Modulus of $\mathrm{La}_{2} \mathrm{NiO}_{4}$. Metals 2021, 11, 1889. https://doi.org/10.3390/ met11121889

Academic Editor: Chang Woo Lee

Received: 29 October 2021

Accepted: 22 November 2021

Published: 24 November 2021

Publisher's Note: MDPI stays neutral with regard to jurisdictional claims in published maps and institutional affiliations.

Copyright: (C) 2021 by the authors. Licensee MDPI, Basel, Switzerland. This article is an open access article distributed under the terms and conditions of the Creative Commons Attribution (CC BY) license (https:/ / creativecommons.org/licenses/by/ $4.0 /)$.
Abstract: Lattice defects significantly affect the mechanical properties of crystalline metal oxides. The materials for the components of solid oxide fuel cells (SOFCs) are no exception, and hence understanding of the interplay between lattice defects and the mechanical properties of components is important to ensure the mechanical stability of SOFCs. Herein, we performed an in situ evaluation of the temperature and $P\left(\mathrm{O}_{2}\right)$ dependence of the elastic moduli of $\mathrm{La}_{2} \mathrm{NiO}_{4}(\mathrm{LN} 214)$, a candidate for the cathode material of SOFCs, using the resonance method to understand the influence of interstitial oxygen on its elastic properties. Above $873 \mathrm{~K}$, both the Young's and shear moduli of LN214 slightly decreased with increasing $P\left(\mathrm{O}_{2}\right)$, suggesting that these elastic moduli are correlated with interstitial oxygen concentration and decreased with increasing interstitial oxygen. We analyzed the influence of interstitial oxygen on the Young's modulus of LN214, based on numerically obtained lattice energy. The $P\left(\mathrm{O}_{2}\right)$ dependence of the Young's modulus of LN214 was found to be essentially explained by variation in the $c$-lattice constant, which was triggered by variation in interstitial oxygen concentration. These findings may contribute to a better understanding of the relationship between lattice defects and mechanical properties, and to the improvement of the mechanical stability of SOFCs.

Keywords: in situ evaluation; elastic modulus; lattice defects; solid oxide fuel cells; $\mathrm{La}_{2} \mathrm{NiO}_{4}$

\section{Introduction}

There is a growing demand for the development of clean and efficient energy conversion technologies that can be an alternative to conventional internal combustion engines. Solid oxide fuel cells (SOFCs) have been regarded as one such technology because of their high power generation efficiency, reduced $\mathrm{CO}_{2}$ emissions, fuel flexibility, and scalability [1-3]. However, further research and development to improve their durability are needed for their full-scale commercialization. In particular, enhancing the mechanical stability of SOFCs is one of the crucial issues to be addressed [4,5]. During operation, SOFC components can be exposed to severe temperature and oxygen chemical potential $\left(\mu_{\mathrm{O}}\right)$ gradients [6-8]. Such temperature and $\mu_{\mathrm{O}}$ gradients can generate immense stress in the components, leading to serious mechanical failures in SOFCs, e.g., cracks and delamination $[9,10]$. To evaluate the stress distribution in SOFC components during operation and effectively suppress mechanical damage, the mechanical properties of SOFC components under operating conditions should be precisely understood.

Many SOFC component materials are known to change their crystal structure and oxygen nonstoichiometry, $\delta$, depending on temperature and oxygen partial pressure $\left(P\left(\mathrm{O}_{2}\right)\right)$, i.e., $\mu_{\mathrm{O}}$, and those changes have a substantial impact on their mechanical properties [11-22]. In particular, the mechanical properties of cathode and interlayer materials are reported to 
be greatly influenced by changes in oxygen nonstoichiometry. For instance, gadoliniumdoped ceria (GDC) is known to change its oxygen nonstoichiometry due to oxygen vacancy formation [19-21]. Changes in the oxygen nonstoichiometry of this material range from a few percent to over a dozen percent depending on dopant concentration, temperature, and $P\left(\mathrm{O}_{2}\right)$. The formation of a relatively large amount of oxygen vacancy is reported to reduce the elastic modulus of GDC by up to several tens of percent. Reduction in the elastic modulus due to the formation of oxygen vacancy has been explained by the expansion of interatomic distance, the decrease in cation valence, and the disappearance of chemical bonds. A similar interplay between oxygen vacancy formation and the elastic modulus is also reported for $\mathrm{La}_{0.6} \mathrm{Sr}_{0.4} \mathrm{Co}_{1-y} \mathrm{Fe}_{y} \mathrm{O}_{3-\delta}$ (LSCF) and its end compositions [22]. Therefore, it is important to understand the relationship between the oxygen nonstoichiometry and elastic properties of SOFC component materials. So far, the influence of variation in oxygen nonstoichiometry due to oxygen vacancy formation on the elastic modulus has been actively investigated. On the other hand, the influence of interstitial oxygen formation on the elastic modulus of SOFC component materials has not been understood well. As in the case of oxygen vacancy formation, interstitial oxygen formation leads to variation in interatomic distance. On the other hand, when interstitial oxygen is formed in the crystal, oxygen ions are placed at normally unoccupied sites in the crystal, and the cation valence as well as the number of chemical bonds are increased, which is in contrast to oxygen vacancy formation. Therefore, interstitial oxygen formation may affect the elastic properties of a material through a different mechanism than oxygen vacancy formation.

In this study, we aimed to understand the influence of interstitial oxygen on the elastic modulus of SOFC components. As a model material, we chose $\mathrm{La}_{2} \mathrm{NiO}_{4+\delta}$ (LN214). LN214 is a Ruddlesden-Popper series oxide $(n=1)$ whose crystal structure is comprised of alternate stacking of a rock salt layer and a perovskite layer $[23,24]$. This material is known to accommodate a comparatively large amount of interstitial oxygen in the rock salt layer [25]. Because of this capability for forming abundant interstitial oxygen, LN214 shows excellent mixed ionic/electronic conductivity, and is hence expected as a candidate for SOFC cathode materials [26-28]. The amount of interstitial oxygen depends on temperature and $P\left(\mathrm{O}_{2}\right)$, and it becomes larger under higher $P\left(\mathrm{O}_{2}\right)$ as well as lower temperatures. To evaluate the influence of interstitial oxygen on the elastic modulus of LN214, the in situ measurements of the elastic modulus at high temperatures and under controlled atmospheres are required. Therefore, we applied a resonance method to evaluate the elastic modulus of LN214. The resonance method is a dynamic measuring method by which elastic moduli are obtained from the resonance frequencies of a sample. The resonance method is a non-destructive method, and hence the temperature and $P\left(\mathrm{O}_{2}\right)$ dependence of the elastic moduli can be systematically measured with high accuracy using a single sample $[13,18,21,22]$. Additionally, this dynamic measuring method can provide the internal friction of the sample, which allows us to evaluate the degree of the non-elastic behavior of the material. Therefore, we performed an in situ evaluation of the temperature and $P\left(\mathrm{O}_{2}\right)$ dependence of the Young's modulus, shear modulus, and internal friction of LN214 using the resonance method to understand the influence of interstitial oxygen on its elastic properties.

\section{Materials and Methods}

\subsection{Sample Preparation}

Powders of LN214 were synthesized by the conventional Pechini method. Nitrate solutions of $\mathrm{La}^{3+}$ and $\mathrm{Ni}^{2+}$, ethylene glycol, and citric acid were mixed in an appropriate ratio and heated to $673 \mathrm{~K}$. The obtained polymeric precursor was calcined at $1273 \mathrm{~K}$. The obtained powders were hydrostatically pressed at $150 \mathrm{MPa}$ and sintered at $1473 \mathrm{~K}$ for $6 \mathrm{~h}$. The heating rate was approximately $390 \mathrm{~K} / \mathrm{h}$, while a slower cooling rate was employed $(106 \mathrm{~K} / \mathrm{h})$ to avoid cracking. The sintered samples of LN214 were characterized by X-ray diffraction (D8 DISCOVER, Bruker AXS, Billerica, MA, USA); it was confirmed that they were single-phase LN214 and had tetragonal symmetry (space group: $14 / \mathrm{mmm}$, the XRD 
pattern of the sample was shown in Figure S1 in the Supplementary Materials), which was consistent with the literature $[23,24]$. The sintered samples were cut into a rectangular shape with dimensions of $45 \times 10 \times 1.5 \mathrm{~mm}^{3}$. The length, width, and thickness of a sample were machined so that respective deviations in parallelism were less than $0.1,0.1$, and $0.02 \mathrm{~mm}$. The surfaces were polished with a diamond paste of $3 \mu \mathrm{m}$. The relative density of the sample was $99 \%$.

\subsection{In Situ Measurements of the Elastic Moduli and Internal Friction}

The elastic moduli (Young's and shear moduli) and the internal friction of sintered compacts of LN214 were evaluated by the resonance method. In this method, the sample is subjected to flexural and torsional oscillations with a frequency of $10^{1}-10^{2} \mathrm{~Hz}$, and the Young's and shear moduli of the sample are determined from the respective resonance frequencies and the sample dimension. The detailed information for this measuring method is described in our previous papers $[13,16,21,22]$. The resonance measurements were performed by using three kinds of equipment (JE, JG, and EGII-HT, Nihon Technoplus Co., Ltd., Osaka, Japan). JE and JG perform freehold resonance measurements and determine the Young's and shear moduli at room temperature, respectively. EGII-HT with an electric furnace and a gas control unit can perform cantilever resonance measurements in the temperature range from room temperature to $1273 \mathrm{~K}$ and under a $P\left(\mathrm{O}_{2}\right)$ of $10^{-1}-10^{-25}$ bar (see also Figure S2 in the Supplementary Materials). Generally, freehold resonance measurements have a higher accuracy than cantilever ones [29]. Therefore, in this work, absolute values of the Young's and shear moduli obtained at room temperature with EGII-HT were calibrated by those obtained with JE and JG. To investigate the temperature dependence of the mechanical properties of LN214, the elastic moduli and internal friction of LN214 were evaluated at every $50 \mathrm{~K}$ while the temperature was elevated from 298 to $1173 \mathrm{~K}$ under a $P\left(\mathrm{O}_{2}\right)$ of $10^{-1}$ bar. The $P\left(\mathrm{O}_{2}\right)$ around the sample was controlled by flowing mixture gases of $\mathrm{O}_{2}$ and $\mathrm{Ar}$, and monitored by an yttria-stabilized zirconia oxygen sensor. At room temperature, 873 , and $1073 \mathrm{~K}$, we evaluated the elastic moduli and the internal friction changing the amplitude of the cantilever oscillation to confirm whether the obtained mechanical properties depended on applied strain or not. Furthermore, at 873, 973, and $1073 \mathrm{~K}$ we evaluated the elastic moduli and the internal friction changing the $P\left(\mathrm{O}_{2}\right)$ from $10^{-4}$ to $10^{-1}$ bar to evaluate the relationship between interstitial oxygen and these mechanical properties. At each measuring temperature and $P\left(\mathrm{O}_{2}\right)$, the measurements were repeated until the measured value reached a constant, i.e., until the sample equilibrated under ambient temperature and $P\left(\mathrm{O}_{2}\right)$. The time required for the equilibration was 5-24 h.

\section{Results and Discussion}

\subsection{Temperature and Oscillation Amplitude Dependence of the Elastic Moduli and Internal Friction of LN214}

We first evaluated the temperature and oscillation amplitude dependence of the elastic moduli and internal friction of LN214. Several SOFC component materials are known to exhibit non-elastic behaviors at high temperatures due to phase transition and/or ferroelasticity. Such non-elastic behaviors have a significant influence on the elastic moduli and internal friction measured by the resonance method, and lead to anomalous temperature dependence of these mechanical properties [12-14,16,22]. Moreover, such non-elastic behaviors make the relationship between stress and strain nonlinear. Thus, the elastic moduli and internal friction of the materials that exhibit the above non-elastic behaviors depend on oscillation amplitude (applied strain) [16]. If LN214 exhibits such behaviors, it is difficult to distinguish whether the change in the elastic moduli is due to the variation in interstitial oxygen concentration or non-elastic behaviors. Therefore, we confirmed whether LN214 shows non-elastic behaviors in the measured temperature and oscillation amplitude ranges.

Figure 1a shows the temperature dependence of the Young's and shear moduli of LN214 under a constant $P\left(\mathrm{O}_{2}\right)$ of $10^{-1}$ bar. The oscillation amplitude was approximately 
$50 \mu \mathrm{m}$. Both the Young's and shear moduli of LN214 gradually and monotonically decreased with increasing temperature. Such temperature dependence was similar to the one of typical refractory oxides, e.g., $\mathrm{Al}_{2} \mathrm{O}_{3}, \mathrm{MgO}$, and $\mathrm{ThO}_{2}$ [30], and the anomalies in the temperature dependence reported for SOFC component materials exhibiting non-elastic behaviors $[11-14,17,22]$ was not observed. The gradual decrease in the Young's modulus with increasing temperature is generally explained by the decrease in the strength of chemical bonds caused by the increase in the interatomic distance with increasing temperature, i.e., thermal expansion [30]. Figure $1 \mathrm{~b}$ shows the temperature dependence of the internal friction of LN214 under the same $P\left(\mathrm{O}_{2}\right)$ and oscillation amplitude. Over the entire temperature range, the magnitude of the internal friction was in the order of $10^{-3}$, which was considerably smaller than that of other SOFC component materials exhibiting the non-elastic behaviors $[13,16,22]$.

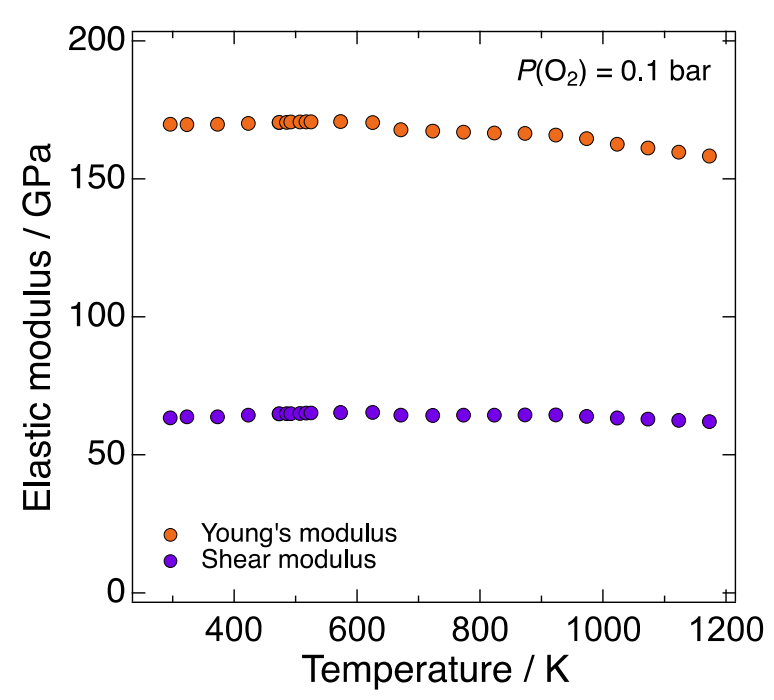

(a)

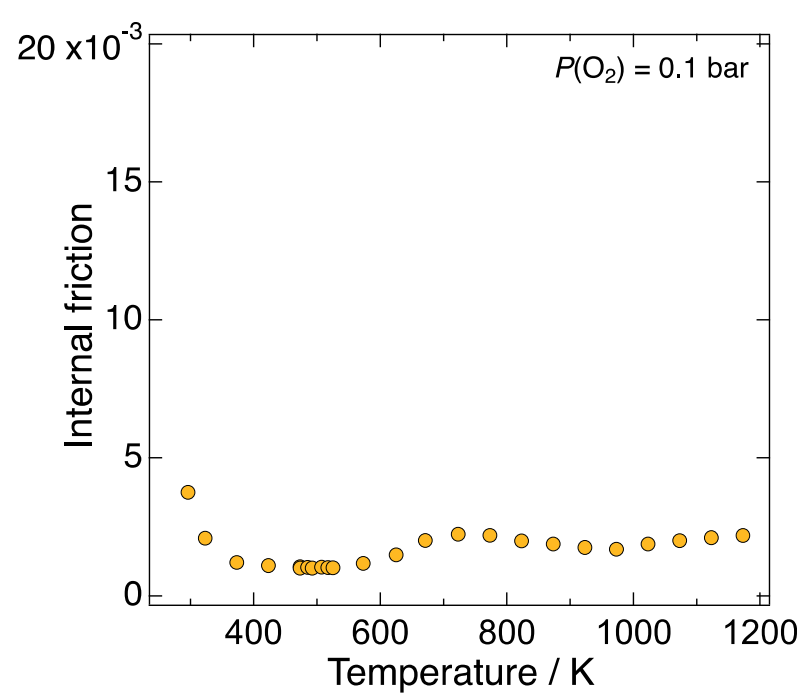

(b)

Figure 1. Temperature dependence of (a) the Young's and shear moduli and (b) the internal friction of LN214 under a constant $P\left(\mathrm{O}_{2}\right)$ of $10^{-1}$ bar. The oscillation amplitude was approximately $50 \mu \mathrm{m}$.

Figure 2a,b show the oscillation amplitude dependence of the Young's modulus and internal friction of LN214, respectively, at room temperature, 873, and $1073 \mathrm{~K}$. At all three temperatures, the Young's modulus was independent of the oscillation amplitude. The magnitude of the internal friction at each temperature remained very low in the measured oscillation amplitude range. These results indicate that the relationship between stress and strain is linear, i.e., LN214 can be regarded to be elastic at least in the measured temperature and oscillation amplitude ranges. Thus, it was confirmed that the change in the elastic moduli could be discussed without considering the above-mentioned non-elastic factors. 


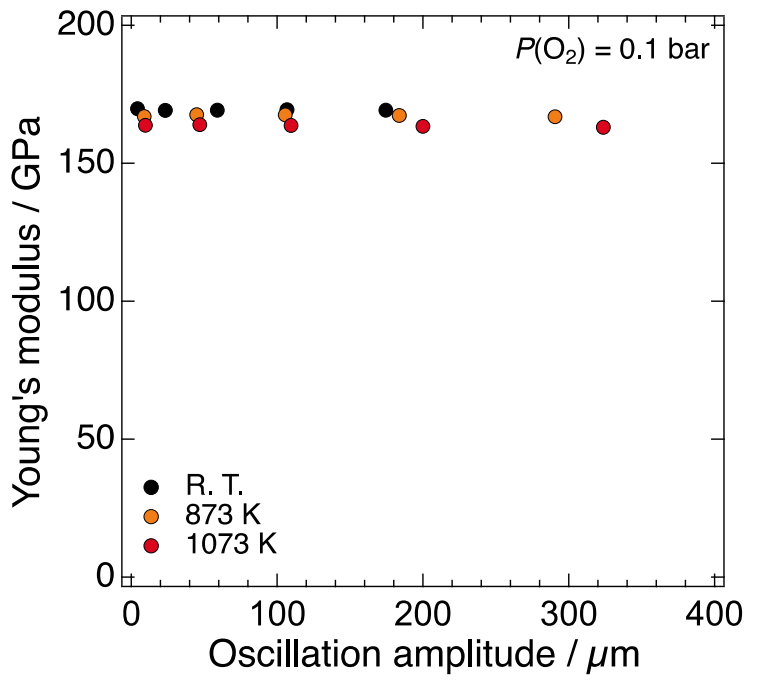

(a)

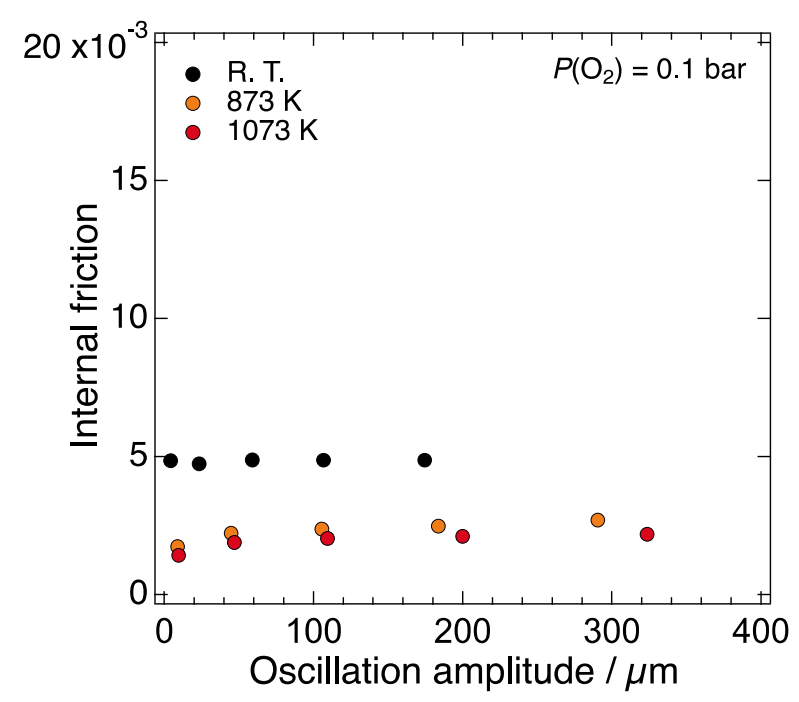

(b)

Figure 2. Oscillation amplitude dependence of (a) the Young's modulus and (b) internal friction of LN214 at room temperature, 873 , and $1073 \mathrm{~K}$ under a constant $P\left(\mathrm{O}_{2}\right)$ of $10^{-1}$ bar.

\section{2. $P\left(\mathrm{O}_{2}\right)$ Dependence of the Elastic Moduli and Internal Friction of LN214}

We then evaluated the $P\left(\mathrm{O}_{2}\right)$ dependence of the elastic moduli and internal friction of LN214. Figure 3a shows the oxygen nonstoichiometry of LN214 as a function of $P\left(\mathrm{O}_{2}\right)$ at 873,973 , and $1073 \mathrm{~K}$ in the $P\left(\mathrm{O}_{2}\right)$ range from $10^{-4}$ to $10^{0}$ bar, which was taken from the literature [25]. Above $873 \mathrm{~K}$ and a $P\left(\mathrm{O}_{2}\right)$ of $10^{-4}$ bar, LN214 shows oxygen excess composition because of interstitial oxygen formation. Interstitial oxygen concentration monotonically increases with increasing $P\left(\mathrm{O}_{2}\right)$ and with decreasing temperature. Considering the above relationship between interstitial oxygen concentration and $P\left(\mathrm{O}_{2}\right)$ /temperature, we measured the elastic moduli and internal friction changing the $P\left(\mathrm{O}_{2}\right)$ from $10^{-4}$ to $10^{-1}$ bar under a constant temperature; thereby, the influence of interstitial oxygen concentration on the elastic moduli can be discussed without the influence of thermal expansion.

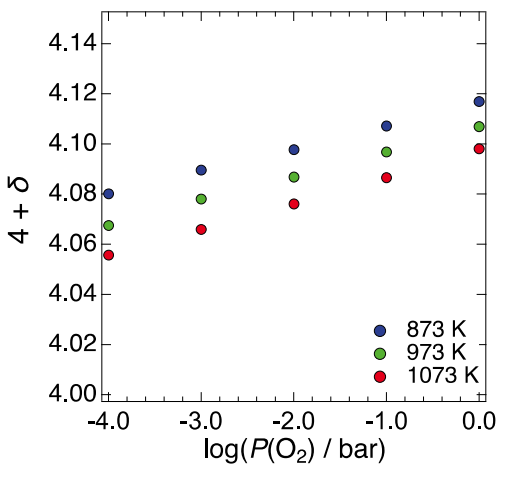

(a)

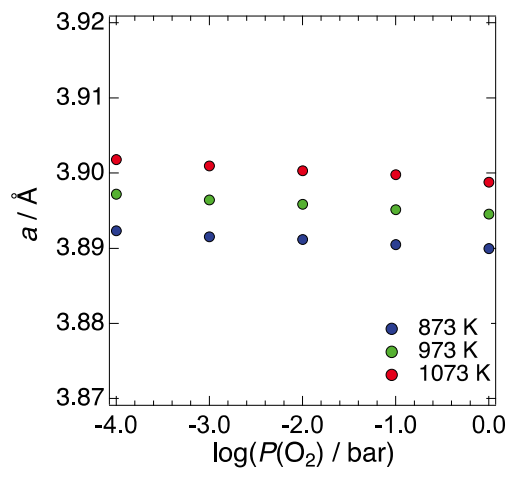

(b)

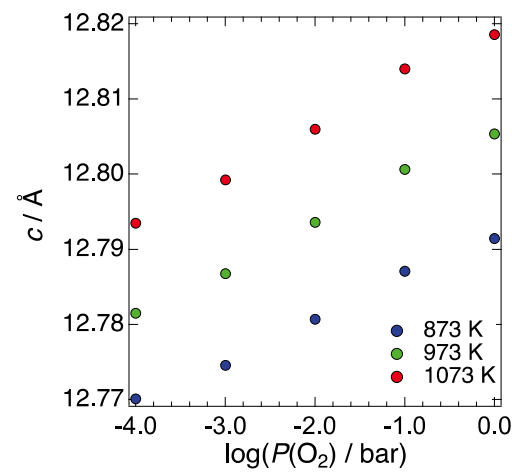

(c)

Figure 3. (a) Oxygen nonstoichiometry, (b) $a$-lattice constant, and (c) $c$-lattice constant of LN214 as a function of $P\left(\mathrm{O}_{2}\right)$ at 873,973 , and $1073 \mathrm{~K}$ in the $P\left(\mathrm{O}_{2}\right)$ range from $10^{-4}$ to $10^{0}$ bar, which was taken from the literature $[24,25]$.

Figure 4a-c show the $P\left(\mathrm{O}_{2}\right)$ dependence of the Young's modulus, shear modulus, and internal friction of LN214, respectively, at 873, 973, and $1073 \mathrm{~K}$. At all three temperatures, both the Young's and shear moduli slightly and monotonically decreased with increasing $P\left(\mathrm{O}_{2}\right)$. These results suggest that the elastic moduli are correlated with interstitial oxygen concentration, and that they decreased with increasing interstitial oxygen. The 
magnitude of the internal friction at each temperature remained very low while changing the $P\left(\mathrm{O}_{2}\right)$, indicating that there is little effect of the non-elastic factors mentioned in the previous section.

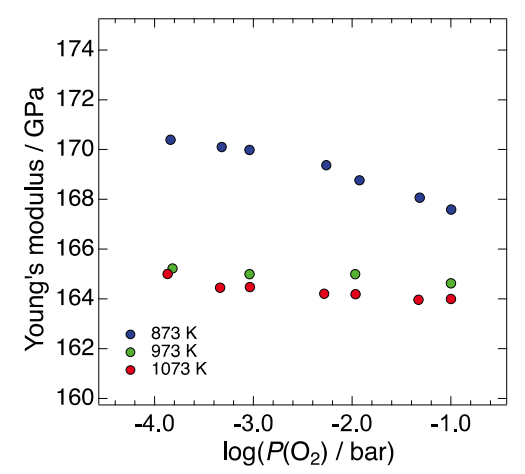

(a)

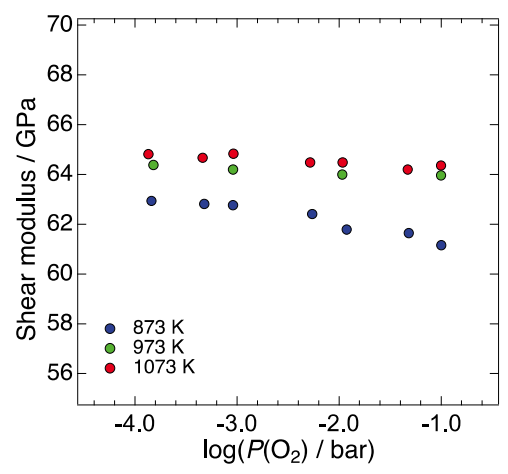

(b)

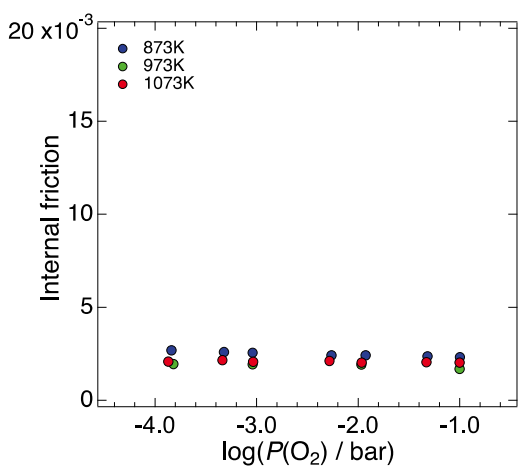

(c)

Figure 4. $P\left(\mathrm{O}_{2}\right)$ dependence of (a) the Young's modulus, (b) shear modulus, and (c) internal friction of LN214, respectively, at 873,973 , and $1073 \mathrm{~K}$.

The change in interstitial oxygen concentration leads to variation in interatomic distance as well as variation in cation valence and the number of chemical bonds. All of these variations can affect the elastic moduli of LN214. In the following section, we discuss which of the variations, which were triggered by interstitial oxygen formation, contributed most to the change in the elastic moduli of LN214.

\subsection{Influence of Interstitial Oxygen Formation on the Elastic Moduli of LN214}

Elastic deformation of an ionic crystal means that the atoms in the crystal are displaced a small distance against the strength of the chemical bonds. On a microscopic scale, therefore, the elasticity of a crystal can be related with the ratio of the strength of the chemical bonds and the change in interatomic distance. Further, the strength of the chemical bonds can be expressed as the first derivative of the lattice energy of the crystal with respect to the interatomic distance. Therefore, the elasticity of a crystal has been discussed based on the relationship between the lattice energy and the interatomic distance [31]. In the field of SOFCs, Duncan et al. [19] and Wang et al. [20] successfully explained the change in the elastic modulus of GDC induced by variation in interatomic distance due to oxygen vacancy formation, assuming the simple Lennard-Jones-type potential. Later, Amezawa et al. [21] modified their approach to deal with not only variation in interatomic distance but also the changes in cation valence and the number of chemical bonds. They also applied the Lennard-Jones-type potential to describe the lattice energy of GDC:

$$
U(r)=-\frac{\mathrm{A}}{r}+\frac{\mathrm{B}}{r^{\mathrm{n}}}
$$

where $r$ is the distance between the nearest-neighbor cations and anions. A is a constant with which to express the electrostatic attractive potential, and is related to the Madelung constant. $\mathrm{B}$ and $\mathrm{n}$ are the constants with which to express the repulsive potential due to the proximity effect. According to the literature, the Young's modulus, E, under equilibrium conditions can be approximated by a second derivative of the potential and the interatomic distance under equilibrium, $r_{\mathrm{eq}}$ :

$$
\left.E \approx \frac{1}{r_{\mathrm{eq}}} \frac{d^{2} U}{d r^{2}}\right|_{r=r_{\mathrm{eq}}}
$$


Moreover, at the equilibrium state:

$$
\left.\frac{d U}{d r}\right|_{r=r_{\mathrm{eq}}}=\frac{\mathrm{A}}{r_{\mathrm{eq}}^{2}}-\mathrm{n} \frac{\mathrm{B}}{r_{\mathrm{eq}}^{\mathrm{n}+1}}=0
$$

From Equations (1)-(3), the Young's modulus can be expressed as follows:

$$
E \approx \frac{1}{2}(\mathrm{n}-1) \frac{\mathrm{A}}{r_{\mathrm{eq}}{ }^{4}}
$$

Therefore, when we describe the Young's modulus, interatomic distance, and the constant $A$ at standard conditions as $E^{0}, r_{\mathrm{eq}}{ }^{0}$, and $\mathrm{A}^{0}$, respectively, the Young's modulus at arbitrary conditions is expressed as [21]:

$$
\frac{E}{E^{0}} \approx \frac{\mathrm{A}}{\mathrm{A}^{0}}\left(\frac{r_{\mathrm{eq}}}{r_{\mathrm{eq}}^{0}}\right)^{-4}
$$

where $r_{\text {eq }}$ and $\mathrm{A}$ are the interatomic distance and the constant $\mathrm{A}$ at arbitrary conditions. The $\left(\mathrm{A} / \mathrm{A}^{0}\right)$ reflects the variations in cation valence and the number of chemical bonds from standard conditions and is a function of oxygen vacancy concentration. For instance, if we take $10 \% \mathrm{Gd}$-doped ceria, $\mathrm{Ce}_{0.9} \mathrm{Gd}_{0.1} \mathrm{O}_{2-\delta}(10 \mathrm{GDC})$, under a $P\left(\mathrm{O}_{2}\right)$ of 1 bar $(\delta=0.05)$ as the standard condition, the ratio of $\mathrm{A}$ of $10 \mathrm{GDC}$ at arbitrary conditions $(2-\delta)$ to $\mathrm{A}^{0}$ becomes [21]:

$$
\frac{\mathrm{A}}{\mathrm{A}^{0}}=\left(\frac{2-\delta}{1.95}\right)^{2}
$$

From Equations (5) and (6), we obtain:

$$
\frac{E}{E^{0}} \approx\left(\frac{2-\delta}{1.95}\right)^{2}\left(\frac{r_{\mathrm{eq}}}{r_{\mathrm{eq}}^{0}}\right)^{-4}
$$

Amezawa et al. reported that the influence of oxygen vacancy on the Young's modulus of 10GDC can be well-described by Equation (7) [21]. However, their approach is not directly applicable to LN214, since Equation (1) assumes that there is only a single kind of cation-anion pair in the crystal. On the other hand, the distance and interaction between the nearest-neighbor cations and anions in LN214 depends on the kind of cations (La or Ni). Therefore, in this study, we modify their approach so that it can be applied to more general ionic oxides. The lattice energy of general ionic oxides can be expressed as:

$$
U=\frac{1}{2} \sum_{i=1}^{N} \sum_{j=1}^{N} \frac{q_{i} q_{j}}{4 \pi \varepsilon_{0} r_{i j}}+\frac{1}{2} \sum_{i=1}^{N} \sum_{j=1}^{N} \frac{\sigma_{i j}}{r_{i j}{ }^{\mathrm{n}}}
$$

where $N, \varepsilon_{0}, q_{i}, r_{i j}$, and $\sigma_{i j}$ are the number of atoms in the crystal, vacuum permittivity, charge of ion $i$, the distance between atoms $i$ and $j$, and a constant to describe the repulsive potential between atoms $I$ and $j$, respectively. The first term on the right-hand side in Equation (8) represents the sum of the electrostatic potentials between the atoms, and the second term represents the sum of the proximity interactions between the atoms. To put Equation (8) in a simple form, we define the average of the distance of the nearest-neighbor cations and anions as the representative interatomic distance in the crystal, $r_{\text {rep: }}$ :

$$
r_{\text {rep }} \equiv \frac{r_{\mathrm{NN} \_1}+r_{\mathrm{NN} \_2}+\cdots+r_{\mathrm{NN} \_p}}{p}
$$

where $r_{\mathrm{NN} \_k}(k=1 \sim p)$ represents the $k$ th one of the $p$ kinds of the nearest cation-anion distances in the crystal. In the case of LN214, Equation (9) represents the average of the 
nearest La-O and Ni-O distances. We express $r_{i j}$ in Equation (8) using the ratio of $r_{i j}$ to $r_{\text {rep, }}$ $\gamma_{i j}$, and $r_{\text {rep }}$ :

$$
r_{i j}=\frac{r_{\text {rep }}}{\gamma_{i j}}
$$

Substituting Equation (10) into Equation (8), we obtain:

$$
U=\frac{1}{2} \sum_{i=1}^{N} \sum_{j=1}^{N} \frac{\gamma_{i j} q_{i} q_{j}}{4 \pi \varepsilon_{0} r_{\text {rep }}}+\frac{1}{2} \sum_{i=1}^{N} \sum_{j=1}^{N} \frac{\gamma_{i j}{ }^{\mathrm{n}} \sigma_{i j}}{r_{\text {rep }}{ }^{\mathrm{n}}}
$$

Here, we define constants $\mathrm{A}^{\prime}$ and $\mathrm{B}^{\prime}$ as:

$$
\begin{aligned}
\mathrm{A}^{\prime} & \equiv-\frac{1}{2} \sum_{i=1}^{N} \sum_{j=1}^{N} \frac{\gamma_{i j} q_{i} q_{j}}{4 \pi \varepsilon_{0}} \\
\mathrm{~B}^{\prime} & \equiv \frac{1}{2} \sum_{i=1}^{N} \sum_{j=1}^{N} \gamma_{i j}{ }^{\mathrm{n}} \sigma_{i j}
\end{aligned}
$$

Using Equations (11)-(13), the lattice energy can be expressed as:

$$
U\left(r_{\text {rep }}\right)=-\frac{\mathrm{A}^{\prime}}{r_{\text {rep }}}+\frac{\mathrm{B}^{\prime}}{r_{\text {rep }}{ }^{\mathrm{n}}}
$$

Equation (14) has a similar form to Equation (1). When we define the representative distance under equilibrium as $r_{\text {rep,eq, }}$ we obtain:

$$
\left.\frac{d U}{d r_{\text {rep }}}\right|_{r_{\text {rep }}=r_{\text {rep }, \text { eq }}}=\frac{\mathrm{A}^{\prime}}{r_{\text {rep,eq }}^{2}}-\mathrm{n} \frac{\mathrm{B}^{\prime}}{r_{\text {rep,eq }}^{\mathrm{n}+1}}=0
$$

As well as Equation (2), a second derivative of Equation (14) with respect to $r_{\text {rep }}$ may be related to the elastic properties of the crystal. For simplicity, we here assume that the Young's modulus can be approximated by a constant multiple of the second derivative of Equation (14):

$$
\left.E \approx \frac{\mathrm{K}}{r_{\text {rep,eq }}} \frac{d^{2} U}{d r_{\text {rep }}^{2}}\right|_{r_{\text {rep }}=r_{\text {rep }, \text { eq }}}
$$

where $\mathrm{K}$ is a constant. Using Equations (15) and (16), the Young's modulus of the crystal can be expressed as:

$$
E \approx \frac{\mathrm{K}(\mathrm{n}-1)}{2} \frac{\mathrm{A}^{\prime}}{r_{\text {rep,eq }}{ }^{4}}
$$

We assume that $\mathrm{K}$ and $\mathrm{n}$ in Equation (17) can be regarded as constants under isothermal conditions and the small variation in interstitial oxygen concentration. Given such assumptions, as in Equation (5), the change in the Young's modulus from standard conditions can be estimated as follows:

$$
\frac{E}{E^{0}} \approx \frac{\mathrm{A}^{\prime}}{\mathrm{A}^{\prime 0}}\left(\frac{r_{\text {rep, eq }}}{r_{\text {rep, eq }}^{0}}\right)^{-4}
$$

where $r^{0}$ rep,eq and $\mathrm{A}^{\prime 0}$ are the representative distance and the constant $\mathrm{A}^{\prime}$ at standard conditions. The relationship between $\mathrm{A}^{\prime}$ and the electrostatic potential in the crystal, $U_{\mathrm{e}}$, is expressed as follows:

$$
U_{\mathrm{e}}=\frac{1}{2} \sum_{i=1}^{N} \sum_{j=1}^{N} \frac{q_{i} q_{j}}{4 \pi \varepsilon_{0} r_{i j}}=-\frac{\mathrm{A}^{\prime}}{r_{\mathrm{rep}}}
$$


If a crystal structure and atomic configurations in the crystal are known, $U_{\mathrm{e}}$ can be numerically calculated using the Ewald summation technique [32]. $r_{\text {rep }}$ can also be calculated from the atomic configurations in the crystal of interest. Therefore, the contribution of the electrostatic potential term to the elastic properties of the crystal, i.e., the second derivative of the electrostatic potential, can be calculated numerically, and hence $\mathrm{A}^{\prime}$ can be numerically obtained by the following equation:

$$
\mathrm{A}^{\prime}=-\left.\frac{r_{\text {rep,eq }}{ }^{3}}{2} \frac{d^{2} U_{\mathrm{e}}}{d r_{\text {rep }}^{2}}\right|_{r_{\text {rep }}=r_{\text {rep }, \text { eq }}}
$$

Using Equations (18) and (20), the change in the Young's modulus from standard conditions can be calculated as follows:

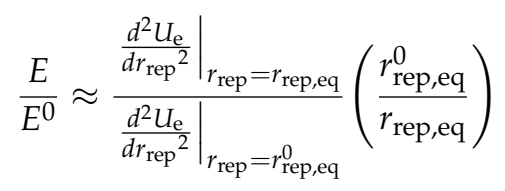

As described above, we consider the change in the Young's modulus under isothermal conditions, i.e., the $P\left(\mathrm{O}_{2}\right)$ dependence of the Young's modulus. Thus, we neglected the contributions from thermal vibration to simplify the discussion, following the literature [21]. In addition, we treated all the chemical bonds in LN214 as pure ionic bonds as a first approximation, although it is reported that the degree of covalence of Ni-O bonds in LN214 is comparatively high $[33,34]$.

In the following, we discuss the $P\left(\mathrm{O}_{2}\right)$ dependence of the Young's modulus of LN214 at constant temperatures, based on Equation (21). As mentioned above, interstitial oxygen concentration monotonically increases with increasing $P\left(\mathrm{O}_{2}\right)$. The changes in interstitial oxygen concentration can lead to various changes in the material. Among them, the elongation and contraction of the lattice are considered to have the most significant influence on the Young's modulus. As mentioned in Section 3.1, the gradual decrease in the Young's modulus with increasing temperature is also explained by the increase in interatomic distance due to thermal expansion and the resulting decrease in bond strength [30]. Moreover, the decrease in the Young's modulus of GDC due to oxygen vacancy formation is also considered to mainly stem from the lattice expansion arising from the change in oxygen nonstoichiometry [19-21]. When interstitial oxygen concentration increases in LN214, the lattice constant of $a$-axis, $a$, decreases, while the lattice constant of $c$-axis, $c$, increases, as shown in Figure 3b,c, respectively, which were taken from the literature [24]. Therefore, using Equation (21), we first simulated to what extent the variations in $a$ and $c$ affected the Young's modulus of LN214, excluding other influential factors such as the variations in cation valence and the number of chemical bonds. To perform such a simulation, we considered a hypothetical case where $a$ and $c$ decrease/increase with increasing $P\left(\mathrm{O}_{2}\right)$, respectively, while the interstitial oxygen concentration remains unchanged. We linearly interpolated the $P\left(\mathrm{O}_{2}\right)$ dependence of $a$ and $c$ of LN214 [24] and calculated the electrostatic potentials at each lattice constant by the Ewald summation technique [32], assuming the crystal structure of LN214 was tetragonal $(\mathrm{I} 4 / \mathrm{mmm})$. We also calculated the representative distance from the nearest $\mathrm{La}-\mathrm{O}$ and $\mathrm{Ni}-\mathrm{O}$ distances at each lattice constant (under each $P\left(\mathrm{O}_{2}\right)$ ), according to Equation (9). In these calculations, interstitial oxygen concentration was fixed to zero, and only the effect of changes in the lattice constants were considered. Figure 5a shows the calculated electrostatic potential of LN214 as a function of the representative distance. The calculated electrostatic potential increased with increasing the representative distance, which was a qualitatively reasonable trend. From the data shown in this figure, we numerically calculated the second derivative of the electrostatic potential (Figure 5b). Using these values and Equation (21), we estimated variation in the Young's modulus from standard conditions at 873,973 , and $1073 \mathrm{~K}$. At each temperature, a $P\left(\mathrm{O}_{2}\right)$ of $10^{-4}$ bar was taken as standard conditions, and the Young's modulus and representative 
distance under a $P\left(\mathrm{O}_{2}\right)$ of $10^{-4}$ bar were used as $E^{0}$, and $r^{0}$ rep,eq, respectively. The black solid lines in Figure 6a-c show the variation in the Young's modulus from the one under a $P\left(\mathrm{O}_{2}\right)$ of $10^{-4}$ bar at 873,973 , and $1073 \mathrm{~K}$, respectively, which were estimated by Equation (21). In the same figures, we show the experimentally observed variation of the Young's modulus for comparison (black circles). At all three temperatures, the estimated values slightly decreased with increasing $P\left(\mathrm{O}_{2}\right)$, which was consistent with the experimental results. At $873 \mathrm{~K}$, the order of magnitude of the variation was in agreement with the experimental results, and at 973 and $1073 \mathrm{~K}$ the estimated variation corresponded reasonably well with the experimental values. This suggests that the variations of the lattice constants have a significant influence on the $P\left(\mathrm{O}_{2}\right)$ dependence of the Young's modulus of LN214.

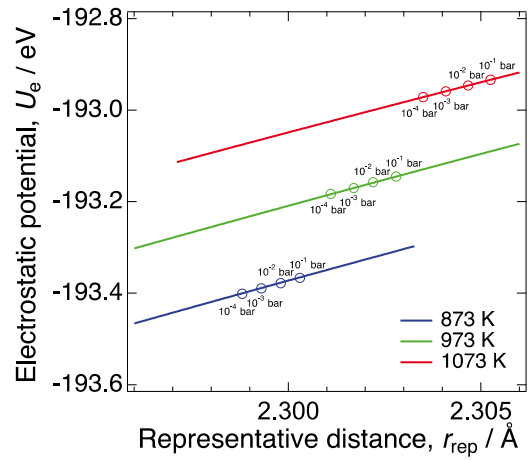

(a)

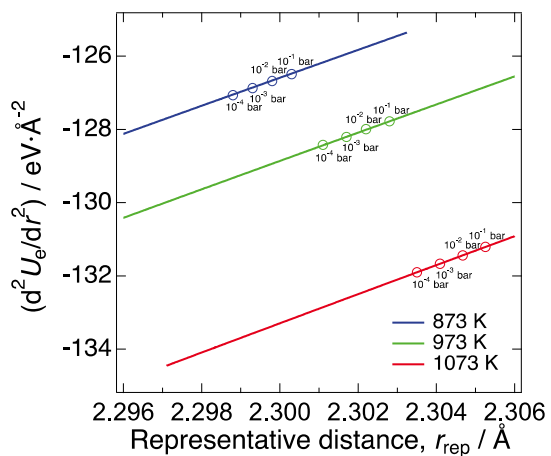

(b)

Figure 5. (a) Electrostatic potential of LN214 as a function of the representative distance at 873, 973, and $1073 \mathrm{~K}$, under the assumption that $a$ - and $c$-lattice constants decrease/increase with increasing $P\left(\mathrm{O}_{2}\right)$, respectively, while the interstitial oxygen concentration remains unchanged. We linearly interpolated the $P\left(\mathrm{O}_{2}\right)$ dependence of $a$ and $c$ of LN214 [24] and calculated the electrostatic potentials at each lattice constant by the Ewald summation technique, assuming the crystal structure of LN214 was tetragonal (I4/mmm). (b) Second derivative of the electrostatic potential of LN214 at 873, 973, and $1073 \mathrm{~K}$, which was numerically calculated using the data shown in Figure 4a.

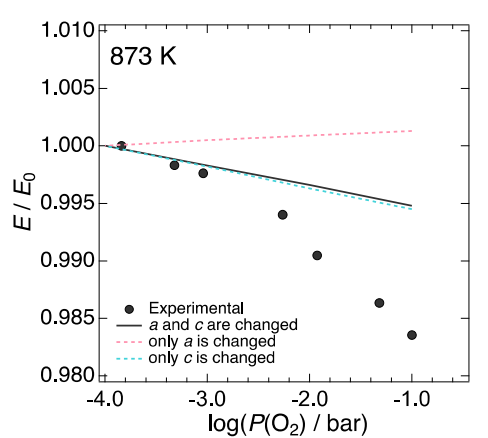

(a)

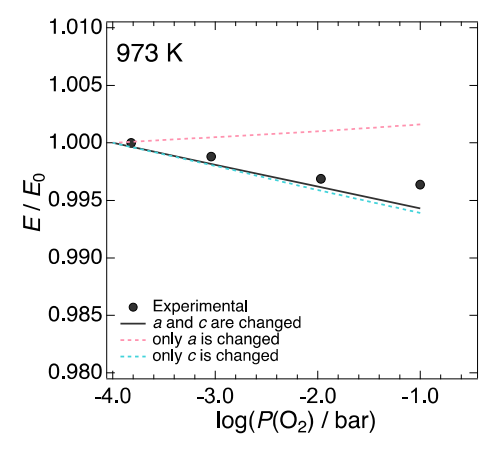

(b)

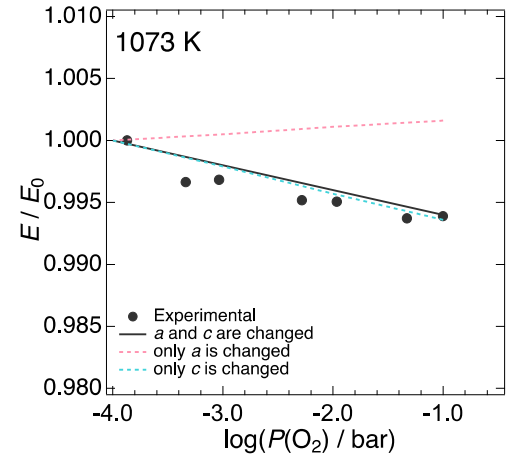

(c)

Figure 6. Change in the Young's modulus of LN214 from the value under a $P\left(\mathrm{O}_{2}\right)$ of $10^{-4}$ bar at (a) 873 , (b) 973 , and (c) 1073 $\mathrm{K}$, respectively. Black circles represent the experimentally observed change. Black lines represent the change in the Young's modulus, which was calculated under the assumption that $a$ - and $c$-lattice constants decreased/increased with increasing $P\left(\mathrm{O}_{2}\right)$, respectively, while the interstitial oxygen concentration remained unchanged. Pink/sky-blue lines represent the change in the Young's modulus, which was calculated under the assumption that only $a$ or $c$ decreased/increased with increasing $P\left(\mathrm{O}_{2}\right)$, respectively, while another lattice constant was fixed to the standard value $\left(a\right.$ or $c$ under a $P\left(\mathrm{O}_{2}\right)$ of $10^{-4}$ bar) and the interstitial oxygen concentration remained unchanged.

As described above, the $a$ of LN214 decreases with increasing $P\left(\mathrm{O}_{2}\right)$; conversely, the $c$ increases [24]. These changes in the lattice constants are expected to have opposite effects 
on the Young's modulus. A decrease in $a$ is presumed to lead to an increase in the Young's modulus, since the contraction of the interatomic distance along the $a$-axis direction can reinforce the bond strength in this direction. Conversely, an increase in $c$ is presumed to decrease the Young's modulus. To separately evaluate the influences of these $a$ and $c$ variations, we considered hypothetical cases where only $a$ or $c$ changes with increasing $P\left(\mathrm{O}_{2}\right)$, while another lattice constant was fixed to the standard value ( $a$ or $c$ under a $P\left(\mathrm{O}_{2}\right)$ of $10^{-4}$ bar). In these calculations, interstitial oxygen concentration was also fixed to zero. The pink/sky-blue lines in Figure $6 a-c$ show the variation in the Young's modulus in hypothetical cases where only $a$ decreases $/ c$ increases with increasing $P\left(\mathrm{O}_{2}\right)$. When only the $a$ variation was considered, the calculated Young's modulus increased slightly with increasing $P\left(\mathrm{O}_{2}\right)$, as expected. On the other hand, the calculated Young's modulus slightly decreased with increasing $P\left(\mathrm{O}_{2}\right)$ in the case where only the $c$ variation was considered. The absolute variation in the Young's modulus when only $c$ was assumed to change was almost comparable to the variation in the experimental values, while the absolute variation in the Young's modulus when only $a$ was assumed to change was smaller than that when only $c$ was assumed to change, as well as the variation of the experimental values. This probably reflects the smaller variation in $a$ compared to that of $c$. When the $P\left(\mathrm{O}_{2}\right)$ is increased from $10^{-4}$ to $10^{-1}$ bar, $a$ decreases by $0.04-0.05 \%$ at $873-1073 \mathrm{~K}$, while $c$ increases by $0.13-0.16 \%$ depending on the temperature [24]. The above discussion suggests that the $P\left(\mathrm{O}_{2}\right)$ dependence of the Young's modulus of LN214 is the result of the combined effect of the contraction of $a$ and the elongation of $c$ with increasing $P\left(\mathrm{O}_{2}\right)$. Since the variation in $c$ with $P\left(\mathrm{O}_{2}\right)$ was much larger than that of $a$, the effect of the $c$ variation was inferred to be dominant in LN214. Therefore, the $P\left(\mathrm{O}_{2}\right)$ dependence of the Young's modulus of LN214 could be reasonably explained by considering the change in the lattice constant, especially the $c$ lattice constant.

So far, we have neglected the change in interstitial oxygen concentration for convenience and have only considered the effect of the changes in lattice constants, which were in reality caused by the change in interstitial oxygen concentration. From now, we discuss to what extent the Young's modulus of LN214 is affected by changes in physical properties triggered by variation in interstitial oxygen concentration, other than variations in lattice constant (e.g., changes in cation valence and the number of chemical bonds). In LN214, the interstitial oxygen ions are randomly distributed in the four equivalent interstitial sites in the rock salt layer. When the $P\left(\mathrm{O}_{2}\right)$ is increased from $10^{-4}$ to $10^{-1}$ bar, the interstitial oxygen concentration of LN214 is known to increase by approximately $\Delta \delta=0.03$ [25]. Such a random distribution of interstitial oxygen and small variation in oxygen nonstoichiometry cannot be rigorously taken into account by the above Ewald summation technique. Thus, we introduced the site occupancy, $\sigma_{i}$, to approximate the electrostatic potential, $U^{\prime}{ }_{\mathrm{e}}$, in the presence of small amounts of interstitial oxygens, as shown in the following equation:

$$
U^{\prime}{ }_{\mathrm{e}}=\frac{1}{2} \sum_{i=1}^{N} \sum_{j=1}^{N} \frac{\left(\sigma_{i} q_{i}\right)\left(\sigma_{j} q_{j}\right)}{4 \pi \varepsilon_{0} r_{i j}}
$$

$\sigma_{i}$ takes the value from 0 to 1: 0 means that no atoms exist on the site of interest while 1 means that the site of interest is fully occupied by atoms. The site occupancy of $\mathrm{La}, \mathrm{Ni}$, and regular $\mathrm{O}$ sites is 1 , and that of interstitial oxygen sites varies depending on oxygen nonstoichiometry. For instance, the oxygen nonstoichiometry of LN214 is $\delta=0.080$ under a $P\left(\mathrm{O}_{2}\right)$ of $10^{-4}$ bar at $873 \mathrm{~K}$, and it increases to $\delta=0.107$ under a $P\left(\mathrm{O}_{2}\right)$ of $10^{-1}$ bar [25]. Accordingly, the $\sigma_{i}$ of interstitial oxygen sites increases from 0.040 to 0.054 . Using Equation (22), we calculated the electrostatic potential of LN214 with interstitial oxygen concentration under a $P\left(\mathrm{O}_{2}\right)$ of $10^{-4}$ bar at each temperature $(\delta=0.080,0.068$, and 0.056 at 873,973 , and $1073 \mathrm{~K}$, respectively [25]) and defined as the standard condition. Further, we calculated the electrostatic potential of LN214 with interstitial oxygen concentration under a $P\left(\mathrm{O}_{2}\right)$ of $10^{-1}$ bar at each temperature $(\delta=0.107,0.097$, and 0.087 at 873,973 , and $1073 \mathrm{~K}$, respectively [25]). From these two potentials, the change in the Young's modulus due to 
variation in interstitial oxygen concentration was calculated based on Equation (22). In these calculations, the lattice constants, $a$ and $c$, were fixed to the values under a $P\left(\mathrm{O}_{2}\right)$ of $10^{-4}$ bar at each temperature to exclude the influence of variations in lattice constant. Figure 7 shows the calculated change in the Young's modulus due to variation in interstitial oxygen concentration (purple bars). At all three temperatures, the calculated Young's modulus increased by approximately $1 \%$ due to the increase in the interstitial oxygen concentration. This suggests that an increase in the interstitial oxygen concentration augments the Young's modulus of LN214. The increase in interstitial oxygen concentration leads to an increase in the valence of $\mathrm{Ni}$ as well as the number of cation-anion bonds. These changes are presumed to reinforce the interatomic bonding strength and hence increase the Young's modulus. However, the experimentally measured Young's modulus decreased by $\sim 0.5 \%$ with increasing interstitial oxygen concentration (with increasing $P\left(\mathrm{O}_{2}\right)$ from $10^{-4}$ to $10^{-1}$ bar), which is contrary to the above calculation (black bars in Figure 7). The reason for such a discrepancy between the experimental and calculated results may be that the approximation using the site occupancy and the estimation of the electrostatic potential were too rough. In the case where the site occupancy is used in the calculation, the electrostatic potential is calculated assuming that the interstitial oxygen sites in LN214 are fully occupied by an element with a charge of $\sigma_{i} q_{i}$. However, the actual situation is different from the above assumption because, in reality, a few oxygen ions with a charge of -2 locally and partially occupy the interstitial oxygen sites. Therefore, although the above calculations are close to reality in terms of the direction of the change in cation valence and the number of chemical bonds, and hence the direction, of variation in the Young's modulus while increasing the interstitial oxygen, the absolute amount of the change in the Young's modulus may be different from reality. In LN214, the increase in interstitial oxygen concentration and the following increase in the $\mathrm{Ni}$ valence and number of chemical bonds probably reinforces the Young's modulus, but the increment may be very small compared to the above calculations and the influence of variations in lattice constant. Therefore, the $P\left(\mathrm{O}_{2}\right)$ dependence of the Young's modulus of LN214 was able to be essentially explained by the change in the lattice constant, especially in the $c$-lattice constant.

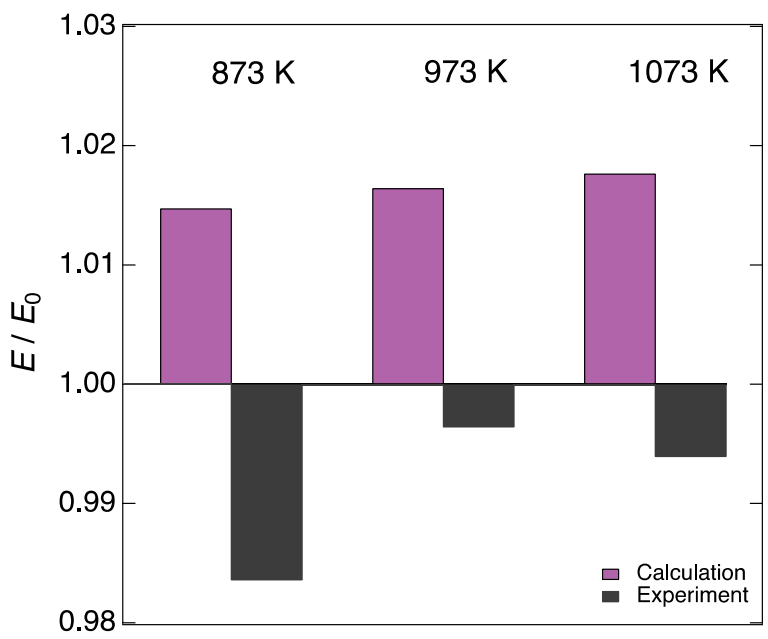

Figure 7. Change in the Young's modulus due to variation in interstitial oxygen concentration that was calculated using Equations (21) and (22) at 873, 973, and $1073 \mathrm{~K}$ (purple bars). These changes were calculated under the assumption that interstitial oxygen concentration of LN214 was changed from the value under a $P\left(\mathrm{O}_{2}\right)$ of $10^{-4}$ bar at each temperature $(\delta=0.080,0.068$, and 0.056 at 873,973 , and $1073 \mathrm{~K}$, respectively [24]) to the one under a $P\left(\mathrm{O}_{2}\right)$ of $10^{-1}$ bar at each temperature $(\delta=0.107,0.097$, and 0.087 at 873,973 , and $1073 \mathrm{~K}$, respectively [24]), while the $a$ - and $c$-lattice constants remained unchanged. Black bars represent the experimentally observed change in the Young's modulus of LN214 when the $P\left(\mathrm{O}_{2}\right)$ was changed from $10^{-4}$ to $10^{-1}$ bar at each temperature. 


\section{Conclusions}

We performed an in situ evaluation of the temperature and $P\left(\mathrm{O}_{2}\right)$ dependence of the Young's modulus, shear modulus, and internal friction of LN214 using the resonance method to understand the influence of interstitial oxygen on its elastic properties. Both the Young's and shear moduli of LN214 gradually and monotonically decreased with increasing temperature. The magnitude of the internal friction of LN214 was small over the entire temperature range. Furthermore, these mechanical properties were independent of oscillation amplitude (applied strain). From these results, it was confirmed that LN214 could be regarded to be elastic in the measured temperature and oscillation amplitude ranges. Above $873 \mathrm{~K}$, both the Young's and shear moduli slightly and monotonically decreased with increasing $P\left(\mathrm{O}_{2}\right)$. These results suggest that the elastic moduli are correlated with the interstitial oxygen concentration, and they decreased with increasing interstitial oxygen. The influence of the interstitial oxygen on the Young's modulus of LN214 was discussed based on numerically obtained lattice energy. It was suggested that the $P\left(\mathrm{O}_{2}\right)$ dependence of the Young's modulus of LN214 was able to be essentially explained by the change in the $c$-lattice constant, which was induced by the variation in interstitial oxygen concentration. On the other hand, the variations in the a-lattice constant, Ni valence, and number of chemical bonds, which were also triggered by the interstitial oxygen concentration variation, were inferred to have less influence on the Young's modulus of LN214. The findings of this study may contribute to a better understanding of the relationship between lattice defects and mechanical properties, and to the improvement of the mechanical stability of SOFCs.

Supplementary Materials: The following are available online at https:/ /www.mdpi.com/article/10 $.3390 /$ met11121889/s1, Figure S1: XRD Pattern of the synthesized $\mathrm{La}_{2} \mathrm{NiO}_{4}$ sample. No impurity peaks were found, and the sample was confirmed to have tetragonal symmetry (space group: $\mathrm{I} 4 / \mathrm{mmm}$ ), Figure S2: Schematic diagram of the in-situ measurements of the Young's modulus, shear modulus and internal friction using the resonance method.

Author Contributions: Conceptualization, Y.K., K.A., K.Y. and T.K.; methodology, Y.K., K.A. and T.K.; software, Y.K.; validation, T.N., K.A., K.Y. and T.K.; formal analysis, Y.K.; investigation, Y.K.; resources, K.A., K.Y. and T.K.; data curation, Y.K.; writing—original draft preparation, Y.K.; writingreview and editing, T.N., K.A., K.Y. and T.K.; visualization, Y.K.; supervision, T.N., K.A., K.Y. and T.K.; project administration, T.K.; funding acquisition, T.K. All authors have read and agreed to the published version of the manuscript.

Funding: This research received no external funding.

Institutional Review Board Statement: Not applicable.

Informed Consent Statement: Not applicable.

Data Availability Statement: The data presented in this study are available upon reasonable request from the corresponding author.

Conflicts of Interest: The authors declare no conflict of interest.

\section{References}

1. Elmer, T.; Worall, M.; Wu, S.; Riffat, S.B. Fuel cell technology for domestic built environment applications: State of-the-art review. Renew. Sustain. Energy Rev. 2015, 42, 913-931. [CrossRef]

2. Mahato, N.; Banerjee, A.; Gupta, A.; Omar, S.; Balani, K. Progress in material selection for solid oxide fuel cell technology: A review. Prog. Mater Sci. 2015, 72, 141-337. [CrossRef]

3. Duan, C.; Huang, J.; Sullivan, N.; O'Hayre, R. Proton-conducting oxides for energy conversion and storage. Appl. Phys. Rev. 2020, 7, 011314. [CrossRef]

4. Singh, P.; Minh, N.Q. Solid oxide fuel cells: Technology status. Int. J. Appl. Ceram. Technol. 2005, 1, 5-15. [CrossRef]

5. Malzbender, J.; Steinbrech, R.W.; Singheiser, L. A review of advanced techniques for characterising SOFC behaviour. Fuel Cells 2009, 9, 785-793. [CrossRef]

6. Atkinson, A. Chemically-induced stresses in gadolinium-doped ceria solid oxide fuel cell electrolytes. Solid State Ion. 1997, 95, 249-258. [CrossRef] 
7. Nakajo, A.; Wuillemin, Z.; Van Herle, J.; Favrat, D. Simulation of thermal stresses in anode-supported solid oxide fuel cell stacks. Part I: Probability of failure of the cells. J. Power Sources 2009, 193, 203-215. [CrossRef]

8. Muramatsu, M.; Yashiro, K.; Kawada, T.; Tarada, K. Numerical simulations of non-stationary distributions of electrochemical potentials in SOFC. Eng. Comput. 2017, 34, 1956-1988. [CrossRef]

9. Sato, K.; Hashida, T.; Yashiro, K.; Yugami, H.; Kawada, T.; Mizusaki, J. Mechanical Damage Evaluation of Solid Oxide Fuel Cells under Simulated Operating Conditions. J. Ceram. Soc. Jpn. 2005, 113, 562-564. [CrossRef]

10. Muramatsu, M.; Sato, M.; Terada, K.; Watanabe, S.; Yashiro, K.; Kawada, T.; Iguchi, F.; Yokokawa, H. Shape deformation analysis of anode-supported solid oxide fuel cell by electro-chemo-mechanical simulation. Solid State Ion. 2018, 319, 194-202. [CrossRef]

11. Lein, H.L.; Andersen, Ø.S.; Vullum, P.E.; Lara-Curzio, E.; Holmestad, R.; Einarsrud, M.-A.; Grande, T. Mechanical properties of mixed conducting $\mathrm{La}_{0.5} \mathrm{Sr}_{0.5} \mathrm{Fe}_{1-\mathrm{x}} \mathrm{Co}_{\mathrm{x}} \mathrm{O}_{3-\delta}(0 \leq \mathrm{x} \leq 1)$ materials. J. Solid State Electrochem. 2006, 10, 635-642. [CrossRef]

12. Huang, B.X.; Malzbender, J.; Steinbrech, R.W.; Singheiser, L. Mechanical properties of $\mathrm{La}_{0.58} \mathrm{Sr}_{0.4} \mathrm{Co}_{0.2} \mathrm{Fe}_{0.8} \mathrm{O}_{3-\delta}$ membranes. Solid State Ionics 2009, 180, 241-245. [CrossRef]

13. Kushi, T.; Sato, K.; Unemoto, A.; Hashimoto, S.; Amezawa, K.; Kawada, T. Elastic modulus and internal friction of SOFC electrolytes at high temperatures under controlled atmospheres. J. Power Sources 2011, 196, 7989-7993. [CrossRef]

14. Araki, W.; Malzbender, J. Ferroelastic deformation of $\mathrm{La}_{0.58} \mathrm{Sr}_{0.4} \mathrm{Co}_{0.2} \mathrm{Fe}_{0.8} \mathrm{O}_{3-\delta}$ under uniaxial compressive loading. J. Eur. Ceram. Soc. 2013, 33, 805-812. [CrossRef]

15. Kimura, Y.; Yashiro, K.; Hashimoto, S.-I.; Kawada, T. Ferroelastic Domain Reorientations and Its Influence on Mechanical Properties of $\mathrm{La}_{0.6} \mathrm{Sr}_{0.4} \mathrm{Co}_{0.2} \mathrm{Fe}_{0.8} \mathrm{O}_{3-\delta}$. J. Electrochem. Soc. 2014, 161, F3079-F3083. [CrossRef]

16. Kimura, Y.; Tolchard, J.; Einarsrud, M.-A.; Grande, T.; Amezawa, K.; Fukuhara, M.; Hashimoto, S.-I.; Kawada, T. Anelastic properties of $\mathrm{La}_{0.6} \mathrm{Sr}_{0.4} \mathrm{Co}_{1-\mathrm{y}} \mathrm{FeyO}_{3-\delta}$ at high temperatures. Solid State Ion. 2014, 262, 337-339. [CrossRef]

17. Xu, Z.C.; Chen, C.C.; Ying, X.N. Oxygen vacancies and their effects on tilting transition in perovskite $\left(\mathrm{La}_{1-x} \mathrm{Pr}_{\mathrm{x}}\right)_{1 / 3} \mathrm{Sr}_{2 / 3} \mathrm{FeO}_{3-\delta}$ investigated by mechanical spectroscopy. Appl. Phys. Lett. 2014, 105, 061905. [CrossRef]

18. Kimura, Y.; Kushi, T.; Unemoto, A.; Amezawa, K.; Kawada, T. Influence of Aging on Mechanical Properties of Yttria-Doped Zirconia. Ceramics 2018, 1, 287-303. [CrossRef]

19. Duncan, K.L.; Wang, Y.; Bishop, S.R.; Ebrahimi, F.; Wachsman, E.D. Role of point defects in the physical properties of fluorite oxides. J. Am. Ceram. Soc. 2006, 89, 3162-3166. [CrossRef]

20. Wang, Y.; Duncan, K.; Wachsman, E.D.; Ebrahimi, F. The effect of oxygen vacancy concentration on the elastic modulus of fluorite-structured oxides. Solid State Ion. 2007, 178, 53-58. [CrossRef]

21. Amezawa, K.; Kushi, T.; Sato, K.; Unemoto, A.; Hashimoto, S.-I.; Kawada, T. Elastic moduli of $\mathrm{Ce}_{0.9} \mathrm{Gd}_{0.1} \mathrm{O}_{2-\delta}$ at high temperatures under controlled atmospheres. Solid State Ion. 2011, 198, 32-38. [CrossRef]

22. Kimura, Y.; Kushi, T.; Hashimoto, S.-I.; Amezawa, K.; Kawada, T. Influences of temperature and oxygen partial pressure on mechanical properties of $\mathrm{La}_{0.6} \mathrm{Sr}_{0.4} \mathrm{Co}_{1-y} \mathrm{FeyO}_{3-\delta}$. J. Am. Ceram. Soc. 2012, 95, 2608-2613. [CrossRef]

23. Takeda, Y.; Kanno, R.; Sakano, M.; Yamamoto, O.; Takano, M.; Bando, Y.; Akinaga, H.; Takita, K.; Goodenough, J.B. Crystal chemistry and physical properties of $\mathrm{La}_{2-x} \mathrm{Sr}_{x} \mathrm{NiO}_{4}(0 \leq \mathrm{x} \leq 1.6)$. Mater. Res. Bull. 1990, 25, 293-306. [CrossRef]

24. Nakamura, T.; Yashiro, K.; Sato, K.; Mizusaki, J. Structural analysis of $\mathrm{La}_{2-x} \mathrm{Sr}_{x} \mathrm{NiO}_{4+\delta}$ by high temperature X-ray diffraction. Solid State Ion. 2010, 181, 292-299. [CrossRef]

25. Nakamura, T.; Yashiro, K.; Sato, K.; Mizusaki, J. Oxygen nonstoichiometry and defect equilibrium in $\mathrm{La}_{2-x} \mathrm{SrxNiO}_{4+\delta} \cdot$ Solid State Ion. 2009, 180, 368-376. [CrossRef]

26. Amow, G.; Skinner, S.J. Recent developments in Ruddlesden-Popper nickelate systems for solid oxide fuel cell cathodes. J. Solid State Electrochem. 2006, 10, 538-546. [CrossRef]

27. Mauvy, F.; Lalanne, C.; Bassat, J.-M.; Grenier, J.-C.; Zhao, H.; Huo, L.; Stevens, P. Electrode properties of $\mathrm{Ln}_{2} \mathrm{NiO}_{4+\delta}\left(\mathrm{Ln}_{=} \mathrm{La}\right.$, Nd, Pr) AC Impedance and DC Polarization Studies. J. Electrochem. Soc. 2006, 153, A1547-A1553. [CrossRef]

28. Tarutin, A.P.; Lyagaeva, J.G.; Medvedev, D.A.; Bi, L.; Yaremchenko, A.A. Recent advances in layered $\mathrm{Ln}_{2} \mathrm{NiO}_{4+\delta}$ nickelates: Fundamentals and prospects of their applications in protonic ceramic fuel and electrolysis cells. J. Mater. Chem. A Mater. Energy Sustain. 2021, 9, 154-195. [CrossRef]

29. Test Method for Young's Modulus of Metallic Materials at Elevated Temperature; JIS Z2280; Japanese Industrial Standards: Tokyo, Japan, 1993.

30. Wachtman, J.B.; Tempt, W.E.; Lam, D.G.; Apstkin, C.S. Exponential Temperature Dependence of Young's Modulus for Several Oxides. Phys. Rev. 1961, 122, 1754-1759. [CrossRef]

31. Galsin, J.S. (Ed.) Chapter 4-Bonding in Solids. In Solid State Physics; Academic Press: Cambridge, MA, USA, 2019 ; pp. 69-91. ISBN 9780128171035.

32. Toukmaji, A.Y.; Board, J.A. Ewald summation techniques in perspective: A survey. Comput. Phys. Commun. 1996, 95, 73-92. [CrossRef]

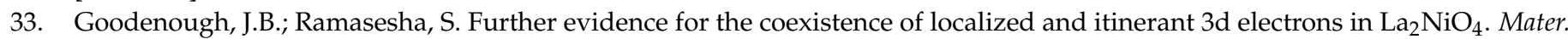
Res. Bull. 1982, 17, 383-390. [CrossRef]

34. Nakamura, T.; Yashiro, K.; Sato, K.; Mizusaki, J. Electronic state of oxygen nonstoichiometric $\mathrm{La}_{2-\mathrm{x}} \mathrm{Sr}_{\mathrm{x}} \mathrm{NiO}_{4+\mathrm{delta}}$ at high temperatures. Phys. Chem. Chem. Phys. 2009, 11, 3055-3062. [CrossRef] [PubMed] 